\title{
PROPRIEDADES TERMOFÍSICAS DE SOLUÇÕES-MODELO SIMILARES A SUCOS - PARTE II
}

Sílvia Cristina Sobottka Rolim de MOURA ", Vitor Correia Lima FRANÇA Áurea Maria Castelo Branco LEAL

\section{RESUMO}

Propriedades termofisicas, densidade e viscosidade de soluções-modelo similares a sucos foram determinadas experimentalmente. Os resultados foram comparados aos preditos por modelos matemáticos (STATISTICA 6.0) e obtidos da literatura em função da sua composição química. Para definição das soluções-modelo, foi realizado um planejamento estrela, mantendo-se fixa a quantidade de ácido (1,5\%) e variando-se a água (82-98,5\%), o carboidrato (0-15\%) e a gordura (0-1,5\%). A densidade foi determinada em picnômetro. A viscosidade foi determinada em viscosimetro Brookfield modelo LVF. A condutividade térmica foi calculada com o conhecimento das propriedades difusividade térmica e calor específico (apresentados na Parte I deste trabalho MOURA [7]) e da densidade. Os resultados de cada propriedade foram analisados através de superficies de respostas. Foram encontrados resultados significativos para as propriedades, mostrando que os modelos encontrados representam as mudanças das propriedades térmicas e fisicas dos sucos, com alterações na composição e na temperatura.

Palavras-chave: condutividade térmica; viscosidade; soluções modelo; sucos.

\section{SUMMARY}

THERMOPHYSICAL PROPERTIES OF MODEL SOLUTIONS SIMILAR TO JUICE - PART II. Thermophysical properties, density and viscosity of model solutions similar to juices were experimentally determined. The results were compared to those predicted by mathematical models (STATISTIC 6.0) and to values mentioned in the literature, according to the chemical composition. A star planning was adopted to define model solutions composition; fixing the acid amount in $1.5 \%$ and varying water $(82-98.5 \%)$, carbohydrate $(0-15 \%)$ and fat $(0-1.5 \%)$. The density was determined by picnometer. The viscosity was determined by Brookfield LVF model viscosimeter. The thermal conductivity was calculated based on thermal diffusivity and specific heat values (presented at the 1 . Part of this paper - MOURA [7]) and density. The results of each property were analyzed by the response surface method. The found results were significant, indicating that the models represent the changes of thermal and physical juices properties, according to their composition and temperature variations.

Keywords: thermal conductivity; viscosity; model solutions; juices.

\section{1 - INTRODUÇÃO}

O dimensionamento dos equipamentos utilizados no processamento de alimentos, principalmente os pasteurizadores e outros que requerem bombeamento do produto, exigem dados precisos das propriedades térmicas dos mesmos (condutividade térmica, difusividade térmica e calor específico) e de como essas propriedades se comportam durante o processo em função da temperatura.

A necessidade de se conhecer as propriedades térmicas com precisão tem levado ao estudo da influência da composição e da temperatura nestas propriedades.

POLLEY, SNYDER \& KOTNOUR [9] publicaram uma coleção de tabelas de propriedades térmicas em diversos alimentos, entre eles carnes, frutas, legumes, leite e cereais. São encontradas nestas tabelas algumas propriedades térmicas de sucos de maçã, cereja, grape, laranja e pêra, porém, estão incompletas.

Recebido para publicação em 21/01/2004. Aceito para publicação em 11/08/2005 (001279).

Centro de Tecnologia de Hortifrutícolas - FRUTHOTEC - /Instituto de Tecnologia de Alimentos - ITAL. Endereco: Avenida Brasil, 2880. CEP.

13073-001, Campinas-SP, Brasil.E-mail: smoura@ital.org.br

Programa de Iniciação Científica CNPq-PIBIC

A quem a correspondência deve ser enviada.
Um trabalho mais específico de determinação de propriedades termofisicas, foi desenvolvido para suco clarificado de maçã, a diversas temperaturas e concentrações [4]. O trabalho apresenta resultados experimentais de determinações de densidade, viscosidade, calor específico e condutividade térmica. Os resultados mostram a grande influência da concentração e temperatura na determinação destas propriedades e modelam os dados em função destas variáveis. No mesmo ano, VIRENDRA, SINGH \& SINGH [13] estudaram a predição da condutividade térmica, obtida por diversos modelos matemáticos, para diversos sucos (maçã, cereja, grape, laranja e morango). Comparando os valores teóricos com valores experimentais o erro encontrado foi menor que $10 \%$.

O conhecimento das propriedades reológicas dos alimentos torna-se necessário para uma série de aplicações como: controle de qualidade, conhecimento da estrutura fisica e, principalmente, controle e dimensionamento de processos industriais.

O efeito da temperatura e da concentração na densidade e viscosidade de suco de maçã foi estudado por BAYINDIRLI [3]. Modelos matemáticos foram obtidos dos dados experimentais apresentando ótima correlação $\left(r^{2}>0,99\right)$. Já em ALVARADO [1], encontra-se a influência da temperatura na viscosidade de sucos de frutas filtrados e do suco de cana-de-açúcar. Os resultados mostram que a viscosidade dos sucos de frutas segue a lei de Arrhenius, apresentando energias de ativação da ordem de $20 \mathrm{~kJ} / \mathrm{g} \cdot \mathrm{mol}$. 
Um trabalho mais recente de predição de propriedades termofísicas aplicada a sucos clarificados, em função da concentração e da temperatura, pode ser encontrado em PEACOCK [8]. O artigo apresenta modelos matemáticos de predição da elevação do ponto de ebulição, densidade, entalpia, calor específico, solubilidade da sacarose, tensão superficial, condutividade térmica e viscosidade. O estudo mostra que existe alta correlação entre as propriedades termofísicas e a concentração e temperaturade produtos fluidos.

Outro trabalho, recentemente apresentado por TELIS-ROMERO et al. [11], obteve resultados significativos na determinação das propriedades termofísicas densidade, difusividade térmica, calor específico e condutividade térmica para suco de laranja não clarificado brasileiro.

É importante destacar o aumento da demanda dos produtos alimentícios no país, trazendo como conseqüência a exigência de modernização, adequação tecnológica e maior qualidade para as indústrias. O cumprimento dessas exigências só acontecerá com maiores informações científicas sobre o processamento de alimentos, o que passa pelo conhecimento das propriedades fisicas para os cálculos dos processos. Hoje, os inúmeros dados de tais propriedades encontrados na literatura para produtos similares não são adequados, na maioria das vezes, para os produtos nacionais. Propriedades termofisicas de grande parte dos produtos brasileiros nem são encontradas na literatura.

A falta de conhecimento de algumas dessas características e de como elas se comportam diante de variações de temperatura pode levar a um processamento inadequado do produto, gerando prejuízos que poderiam ser facilmente evitados. A indústria de sucos tropicais no Brasil sofre com a falta de dados que sirvam de base para estudos de dimensionamento de processamentos térmicos. Assim, o objetivo do presente trabalho foi determinar experimentalmente a densidade e a viscosidade e, com o conhecimento prévio do calor específico e da difusividade térmica, calcular a condutividade térmica de soluções- modelo similares a sucos, na faixa de temperatura de 10 a $80^{\circ} \mathrm{C}$, estudando a influência da composição química e da temperatura nas propriedades fisicas dos produtos. Foi feita ainda uma comparação entre os dados experimentais e valores calculados obtidos de modelos matemáticos encontrados na literatura.

\section{2 - MATERIAL E MÉTODOS}

\section{1 - Materiais}

As soluções-modelo foram preparadas seguindo uma distribuição de componentes dada por um tratamento estatístico [6], tentando-se simular um suco de fruta. Foram variados os teores de água (82 a 98,5\%,), carboidrato ( 0 a $15 \%$ ), gordura ( 0 a $1,77 \%$ ), mantendo-se fixa a quantidade de ácido em 1,5\%. Para estabilizar a emulsão, utilizou-se goma xantana a $0,2 \%$.

\section{2 - Métodos}

\subsection{1 - Densidade $(\rho)$}

Determinada em triplicata, a $50^{\circ} \mathrm{C}$, pelo método de deslocamento de fluidos em picnômetro, segundo norma $\mathrm{n}^{\mathrm{o}} 985.19$ da AOAC [13]. Os picnômetros foram previamente calibrados com água destilada e os dados coletados em triplicata.

\subsection{2 - Condutividade térmica (k)}

Determinada após o conhecimento da difusividade térmica, densidade e do calor específico do produto, usando a relação (I):

$k=\rho \cdot C p \cdot \alpha$

Onde:

$\mathrm{k}\left(\mathrm{kcal} / \mathrm{m} .{ }^{\circ} \mathrm{C} . \mathrm{h}\right)$

$\rho=$ densidade $\left(\mathrm{kg} / \mathrm{m}^{3}\right)$

$\mathrm{Cp}=$ calor específico $\left(\mathrm{kcal} / \mathrm{kg}^{\circ} \mathrm{C}\right)$

$\alpha=$ difusividade térmica $\left(\mathrm{m}^{2} / \mathrm{h}\right)$

\subsection{3 - Viscosidade $(\eta)$}

Determinada em triplicata, utilizando um viscosimetro Brookfield LVF spindle \#1 à $60 \mathrm{rpm}$, conforme recomendações de VAN WAZER et al. [12].

\subsection{4 - Delineamento experimental e avaliação dos resultados}

A análise de superficie de resposta foi feita para otimizar as propriedades termofísicas citadas. Para tanto utilizou-se o Planejamento Estrela [2], o qual consiste em um método rotacional central, onde $\mathrm{X}$ e $\mathrm{X}$ são variáveis codificadas de acordo com o planejamento. Todos os dados foram coletados em triplicata. Para obtenção da superficie de resposta foi utilizado o programa STATISTICA 6.0 .

O modelo experimental de segunda ordem (II) descreve as superficies de resposta para as variáveis densidade, viscosidade e condutividade térmica.

$Y=B_{s}+B_{v} X_{s}+B_{2} X_{c}^{2}+B_{s} X_{2}+B_{s} X_{2}^{2}+B_{s} X_{i} X_{2}$

onde $\mathrm{Y}$ é a resposta medida experimentalmente para as

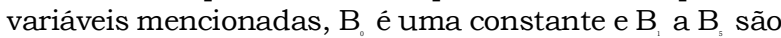
coeficientes numéricos para as variáveis codificadas $\mathrm{X}$ e $\mathrm{X}$.

$$
X_{1}=\frac{\% \text { óleo }}{\% \text { água }+\% \text { carboidrato }} \quad X_{2}=\frac{\% \text { carboidrato }}{\% \text { água }}
$$

O método de superficie de resposta determina as condições ótimas através da combinação entre "design" experimental especial com modelagem matemática, utilizando-se para tanto equações polinomiais de segunda ordem. Neste estudo adotou-se o Planejamento Estrela com três repetições do ponto central, a fim de analisar as propriedades especificadas anteriormente, variando-se a 
composição de água ( $82 \%$ a $98,5 \%)$, carboidrato (0 a $15 \%$ ) e gordura ( 0 a $1,77 \%$ ) e mantendo-se fixa a concentração de ácido a 1,5\%. Para cada propriedade física foram realizados 11 ensaios, seguindo o planejamento inicial para a viabilização da superficie de resposta. O planejamento encontra-se na Tabela 1.

TABELA 1 - Composição dos ensaios utilizada para determinar as propriedades fisicas

\begin{tabular}{|c|c|c|c|c|c|c|c|}
\hline Ensaio & $X_{1}$ & $\mathrm{X}_{2}$ & $\begin{array}{c}\text { Água } \\
(\%)\end{array}$ & $\begin{array}{c}\text { Carboidrato } \\
(\%)\end{array}$ & $\begin{array}{c}\text { Óleo } \\
\%)\end{array}$ & $\begin{array}{c}\text { Ácido } \\
(\%)\end{array}$ & $\begin{array}{c}\text { Total } \\
(\%)\end{array}$ \\
\hline 1 & -1 & -1 & 95,63 & 2,58 & 0,29 & 1,50 & 100 \\
\hline 2 & 1 & -1 & 94,40 & 2,55 & 1,55 & 1,50 & 100 \\
\hline 3 & -1 & 1 & 84,97 & 13,24 & 0,29 & 1,50 & 100 \\
\hline 4 & 1 & 1 & 83,87 & 13,08 & 1,55 & 1,50 & 100 \\
\hline 5 & 0 & 0 & 89,47 & 8,14 & 0,89 & 1,50 & 100 \\
\hline 6 & 0 & 0 & 89,47 & 8,14 & 0,89 & 1,50 & 100 \\
\hline 7 & 0 & 0 & 89,47 & 8,14 & 0,89 & 1,50 & 100 \\
\hline 8 & $-1,4142$ & 0 & 90,28 & 8,22 & 0,00 & 1,50 & 100 \\
\hline 9 & 0 & 1,4142 & 82,51 & 15,10 & 0,89 & 1,50 & 100 \\
\hline 10 & 1,4142 & 0 & 88,67 & 8,06 & 1,77 & 1,50 & 100 \\
\hline 11 & 0 & $-1,4142$ & 97,61 & 0,00 & 0,89 & 1,50 & 100 \\
\hline
\end{tabular}

Os valores obtidos experimentalmente para as propriedades termofísicas serão comparados com os obtidos através de modelos matemáticos encontrados na literatura.

\section{3 - RESULTADOS E DISCUSSÃO}

Os resultados médios de $\mathrm{pH}$, sólidos solúveis, densidade, calor específico e difusividade térmica, obtidos na Parte I do trabalho [7], e a condutividade térmica, calculada através da equação (I), estão dispostos na Tabela 2.

TABELA 2 - Resultados médios das propriedades: Sólidos solúveis, $\mathrm{pH}$, densidade, calor específico, difusividade e condutividade térmicas, sendo o três últimos a $50^{\circ} \mathrm{C}$

\begin{tabular}{ccccccc}
\hline Ensaio & $\begin{array}{c}\text { Sólidos } \\
\text { solúveis }\end{array}$ & $\mathrm{pH}$ & $\begin{array}{c}\mathrm{Cp} \\
\left(\mathrm{kcal} / \mathrm{kg}^{\circ} \mathrm{C}\right)\end{array}$ & $\begin{array}{c}\rho\left(\mathrm{kg} / \mathrm{m}^{3}\right) \\
\times 10^{-3}\end{array}$ & $\begin{array}{c}\alpha\left(\mathrm{m}^{2} / \mathrm{h}\right) \\
\times 10^{4}\end{array}$ & $\begin{array}{c}\mathrm{k} \\
\left(\mathrm{kcal} / \mathrm{m} .{ }^{\circ} \mathrm{C} . \mathrm{h}\right)\end{array}$ \\
\hline 1 & 5,0 & 2,48 & 0,8884 & 1,0095 & 3,8041 & 0,3412 \\
2 & 4,5 & 2,50 & 0,9903 & 1,0059 & 5,5253 & 0,5504 \\
3 & 15,5 & 2,36 & 0,8098 & 1,0534 & 3,5812 & 0,3055 \\
4 & 15,0 & 2,45 & 0,9359 & 1,0382 & 3,8621 & 0,3753 \\
5 & 9,0 & 2,40 & 0,8534 & 1,0213 & 4,0681 & 0,3546 \\
6 & 9,0 & 2,40 & 0,8424 & 1,0213 & 3,8718 & 0,3331 \\
7 & 9,0 & 2,40 & 0,8419 & 1,0213 & 4,1411 & 0,3561 \\
8 & 10,0 & 2,27 & 0,8380 & 1,0323 & 2,8737 & 0,2486 \\
9 & 17,0 & 2,23 & 0,8664 & 1,0413 & 3,5268 & 0,3182 \\
10 & 9,6 & 2,48 & 0,9283 & 1,0309 & 4,3510 & 0,4164 \\
11 & 2,0 & 2,31 & 0,9755 & 0,9939 & 5,1325 & 0,4976 \\
\hline
\end{tabular}

Os dados da Tabela 2 possibilitaram construir os gráficos de superficie de resposta para as propriedades termofisicas: densidade (Figura 1), viscosidade (Figura 2) e condutividade térmica (Figura 3), em função das variáveis $\mathrm{X}_{i}$ e $\mathrm{X}$, que serão apresentados mais adiante.

Através do programa Statistica 6.0 calcularam-se os coeficientes das equações geradas pelo modelo de superficie de resposta, bem como seus limites de confiança de $95 \%$.

\section{1 - Densidade $(\rho)$}

A análise de variância avalia o quanto o modelo de superficie de resposta representa à variação dos dados observados. Para densidade, o modelo representa $81,1 \%$ da variação dos valores observados, em nivel de significância de $5 \%$. O fator de ajuste para densidade foi de aproxi- madamente 0,02 , portanto abaixo do valor nominal de 0,05 (95\% de probabilidade). Isto significa que, apesar da equação ter um coeficiente de determinação $\left(\mathrm{r}^{2}=0,89\right)$ bom, não é o melhor ajuste possivel. O modelo foi descrito com um intervalo de confiança (95\%) de cada constante obtida.

é:

O modelo de segunda ordem para densidade (DENS)

DENS $=(1025,29 \pm 3,74)-(3,33 \pm 2,30) X_{1}+(2,87 \pm 1,78) X_{1}^{2}-(2,57 \pm 0,98) X_{2}^{2}-(2,90 \pm 3,99) X_{1} X_{2}^{2}$

onde as variáveis $\mathrm{X}$ e $\mathrm{X}$ são codificadas conforme Tabela 1 .

De acordo com a Figura 1, quanto maior o valor de $\mathrm{X}$, menor o valor da densidade. Isto é fácil de visualizar, uma vez que os coeficientes $B$ e $B$, os quais estão atrelados à variável $\mathrm{X}_{1}$, são negativos. Por outro lado, o efeito negativo de $\mathrm{X}$ na densidade torna-se claro para valores altos de $\mathrm{X}$

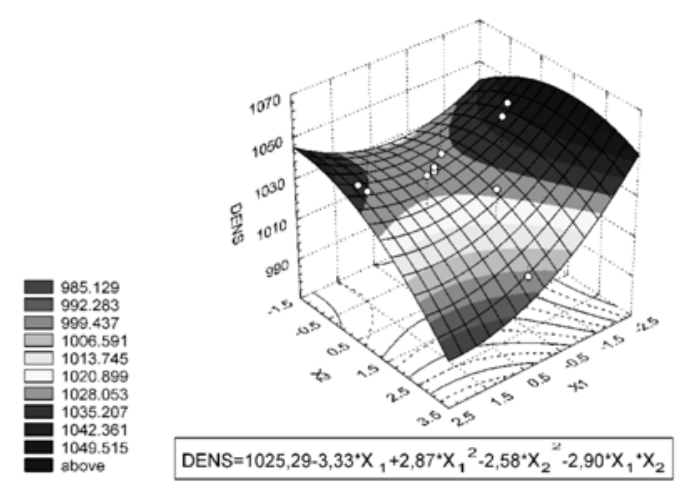

FIGURA 1 - Densidade em função de X e X

Os valores encontrados experimentalmente foram comparados aos do modelo proposto por PEACOCK [8].

$\rho=1000 \cdot\left[1+B \cdot\left(\frac{B+200}{54000}\right)\right]\left[1-0,036 \cdot\left(\frac{T-20}{160-T}\right)\right]$

onde:

$\rho=$ Densidade $\left(\mathrm{kg} / \mathrm{m}^{3}\right)$

$\mathrm{B}=$ Concentração de sólidos solúveis ( $\left.{ }^{\circ} \mathrm{Brix}\right)$

$\mathrm{T}=$ Temperatura $\left({ }^{\circ} \mathrm{C}\right)$

Pela análise dos valores da Tabela 3 percebe-se que o erro é muito pequeno, no máximo 3,06\% (ensaio 9).

TABELA 3 - Erro experimental para densidade, segundo equação (IV)

\begin{tabular}{cccc}
\hline & \multicolumn{2}{c}{$\mathrm{kg} / \mathrm{m}^{3}$} & $\%$ \\
\cline { 2 - 4 } Ensaio & $\rho_{\exp }$ & $\rho_{\text {equaçào IV }}$ & Erroequaçăo IV $^{*}$ \\
\hline 1 & 1009,5 & 1009,0 & 0,05 \\
2 & 1005,9 & 1021,6 & 1,56 \\
3 & 1053,4 & 1066,6 & 1,26 \\
4 & 1038,2 & 1064,5 & 2,53 \\
5 & 1021,3 & 1039,5 & 1,78 \\
6 & 1021,3 & 1039,5 & 1,78 \\
7 & 1021,3 & 1039,5 & 1,78 \\
8 & 1032,3 & 1043,6 & 1,09 \\
9 & 1041,3 & 1073,1 & 3,06 \\
10 & 1030,9 & 1041,9 & 1,07 \\
11 & 993,9 & 1012,0 & 1,82 \\
\hline Erro = (valor teórico - valor experimental)/ valor experimental x 10
\end{tabular}




\section{2 - Viscosidade $(\eta)$}

A viscosidade é uma propriedade que varia muito com a temperatura. Foram realizadas medidas a três temperaturas: $6^{\circ} \mathrm{C}, 30^{\circ} \mathrm{C}$ e $50 \hat{\mathrm{E}}^{\circ} \mathrm{C}$, que encontram-se na Tabela 4.

TABELA 4 - Viscosidade cinemática dos ensaios 1 a 11 a $6^{\circ} \mathrm{C}$, $30^{\circ} \mathrm{C}$ e $50^{\circ} \mathrm{C}$

\begin{tabular}{cccc}
\hline & \multicolumn{3}{c}{$\eta(\mathrm{cP})$} \\
\cline { 2 - 4 } Ensaio & $\mathrm{T}\left(6^{\circ} \mathrm{C}\right)$ & $\mathrm{T}\left(30^{\circ} \mathrm{C}\right)$ & $\mathrm{T}\left(50^{\circ} \mathrm{C}\right)$ \\
\hline 1 & 58,45 & 33,40 & 20,70 \\
2 & 51,30 & 28,50 & 17,50 \\
3 & 68,40 & 38,00 & 23,50 \\
4 & 65,10 & 35,90 & 21,80 \\
5 & 62,60 & 34,45 & 20,60 \\
6 & 62,70 & 34,50 & 20,60 \\
7 & 62,75 & 34,50 & 20,50 \\
8 & 54,00 & 30,70 & 18,80 \\
9 & 57,60 & 33,80 & 20,50 \\
10 & 58,45 & 32,35 & 19,60 \\
11 & 42,10 & 22,85 & 13,90 \\
\hline
\end{tabular}

A resposta encontrada para viscosidade representa apenas $60,7 \%$ da variação dos valores observados, em nível de significância de $5 \%$. O fator de ajuste para viscosidade foi de aproximadamente 0,003 , portanto abaixo do valor nominal de 0,05. Desta maneira, não se pode afirmar que o modelo encontrado seja o melhor, devendo-se assim ocorrer testes para verificar outras modelagens matemáticas. O modelo foi descrito com um intervalo de confiança $(95 \%)$ de cada constante obtida. O coeficiente de determinação ( $r^{2}$ ) do modelo foi de 0,76 .

O modelo de segunda ordem para viscosidade (VISC) é:

VISC $=(22,09 \pm 0,36)-(0,93 \pm 0,22) X_{1}-[0,17 \pm 0,17] X_{1}^{2}+(2,63 \pm 0,35) X_{2}-(1,57 \pm 0,17) X_{2}^{2}$

De acordo com a Figura 2, o aumento de X ocasiona o aumento da viscosidade. Isto equivale a dizer que, em sucos o aumento de carboidrato interfere positivamente no valor desta propriedade física.

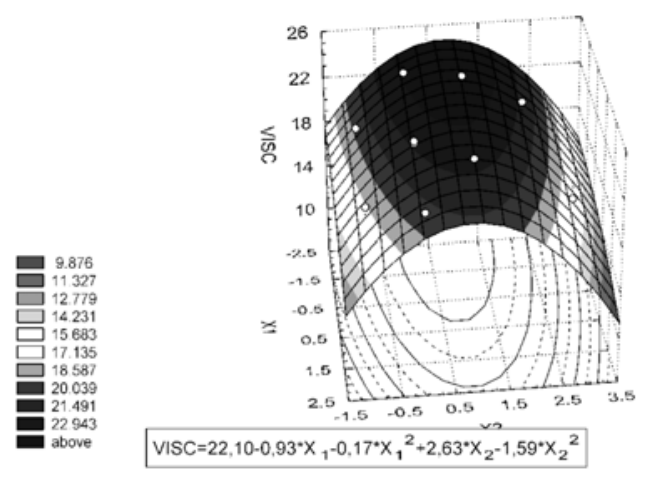

FIGURA 2 - Viscosidade em função de $\mathrm{X}_{1}$ e $\mathrm{X}_{2}$

Os dados encontrados em literatura para se estimar a viscosidade de sucos são bem específicos. Os poucos trabalhos que abordam esta propriedade fisica referemse a sua variação em função da temperatura utilizandose da relação de Arrhenius: $\eta=\eta \cdot \exp \left(\frac{E a}{R . T}\right)$

onde:

$\eta=$ viscosidade,$(\mathrm{cP})$

$\eta_{0}=$ fator pré-exponencial, $(\mathrm{cP})$

$\mathrm{E}=$ Energia de ativação de fluxo, $(\mathrm{kcal} / \mathrm{gmol})$

$\mathrm{R}=$ Constante dos gases, $\left(1,987.10^{-3} \mathrm{kcal} / \mathrm{gmol} .{ }^{\circ} \mathrm{K}\right)$

Plotando-se um gráfico de $\ln \eta$ versus $1 /$ T pode-se determinar os valores de $\eta$ e de $E$, uma vez que $\ln \eta$ e $E / R$ são os coeficientes linear e angular da reta, respectivamente.

Utilizando-se a relação de Arrhenius, os valores encontrados para a viscosidade nas três temperaturas medidas foram inferiores a $5 \%$. Apesar da viscosidade ter sido medida em apenas 3 pontos, a regressão linear usada para aplicar a relação de Arrhenius apresentou um bom $r^{2}$ (Tabela 5), sendo então uma boa relação para a viscosidade nesta faixa de temperatura.

TABELA 5 - Erro experimental para viscosidade, segundo equação (VI), a $50^{\circ} \mathrm{C}$

\begin{tabular}{cccccc}
\hline & & & \multicolumn{2}{c}{$\eta$} & $\%$ \\
\cline { 4 - 6 } Ensaio & Equação & $\mathrm{r}^{2}$ & $\eta_{\exp }$ & $\eta_{\text {equaçảo VI }}$ & Erroequaçào VI \\
\hline 1 & $\mathrm{y}=2116,33 \mathrm{x}-3,5051$ & 0,998 & 20,70 & 21,05 & 1,70 \\
2 & $\mathrm{y}=2194,44 \mathrm{x}-3,9173$ & 0,9992 & 17,50 & 17,75 & 1,45 \\
3 & $\mathrm{y}=2180,78 \mathrm{x}-3,5818$ & 0,9993 & 23,50 & 23,80 & 1,29 \\
4 & $\mathrm{y}=2231,64 \mathrm{x}-3,8115$ & 0,9991 & 21,80 & 22,15 & 1,59 \\
5 & $\mathrm{y}=2265,62 \mathrm{x}-3,9702$ & 0,998 & 20,60 & 20,99 & 1,90 \\
6 & $\mathrm{y}=2268,71 \mathrm{x}-3,9795$ & 0,998 & 20,60 & 21,00 & 1,93 \\
7 & $\mathrm{y}=2279,76 \mathrm{x}-4,0176$ & 0,998 & 20,50 & 20,92 & 2,03 \\
8 & $\mathrm{y}=2150,26 \mathrm{x}-3,7046$ & 0,998 & 18,80 & 19,16 & 1,89 \\
9 & $\mathrm{y}=2100,99 \mathrm{x}-3,4582$ & 0,997 & 20,50 & 21,04 & 2,63 \\
10 & $\mathrm{y}=2228,20 \mathrm{x}-3,9061$ & 0,998 & 19,60 & 19,93 & 1,70 \\
11 & $\mathrm{y}=2262,30 \mathrm{x}-4,3594$ & 0,9994 & 13,90 & 14,08 & 1,29 \\
\hline Erro = (valor teórico-valor experimental)/ valor experimental x 100
\end{tabular}

\section{3 - Condutividade térmica (k)}

Uma vez determinados os valores de difusividade térmica, densidade e calor específico, podem-se calcular os valores de $\mathrm{k}$ para os 11 ensaios. Estes valores estão listados na Tabela 2.

A resposta encontrada para condutividade térmica representa $86,2 \%$ da variação dos valores observados, em nivel de significância de $5 \%$. O fator de ajuste para condutividade térmica foi de aproximadamente 0,12 , portanto acima do valor nominal de 0,05, indicando que o modelo de segunda ordem aplicado para condutividade térmica é apropriado para justificar a variação desta propriedade térmica com a variação de $\mathrm{X}$ e $\mathrm{X}$. O modelo foi descrito com um intervalo de confiança (95\%) de cada constante obtida. O coeficiente de determinação $\left(\mathrm{r}^{2}\right)$ do modelo foi de 0,89 .

O modelo de segunda ordem para condutividade térmica (COND) é:

$C O N D=(0,352 \pm 0,028)+(0,013 \pm 0,009) X_{2}^{2}-(0,035 \pm 0,040) X_{1} X_{2}$

(VII) 
De acordo com a Figura 3, quanto maior o valor de X menor se torna o valor da condutividade térmica. Sendo assim, da mesma forma que o aumento de $\mathrm{X}$ diminui o valor da difusividade térmica, também diminui o valor da condutividade térmica.
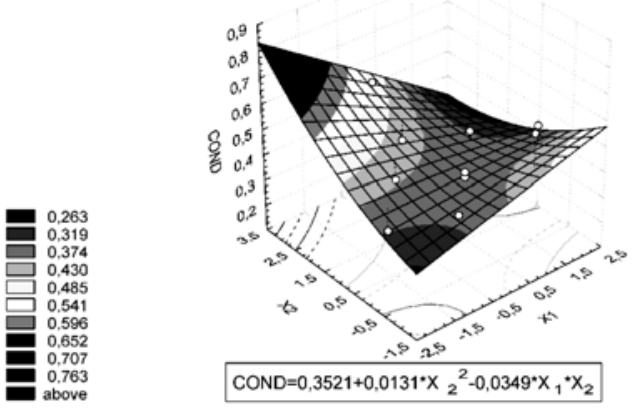

FIGURA 3 - Condutividade térmica em função de $\mathrm{X}_{\mathrm{r}}$ e X

Os valores encontrados experimentalmente foram comparados aos modelos propostos por KOLAROV \& GROMOV [5] e SWEAT [10]:

- Modelo KOLAROV and GROMOV

$k-0,140+0,42 . w \quad\left(\mathrm{~W} / \mathrm{m}^{\circ} \mathrm{C}\right)$

onde:

$\mathrm{w}=$ umidade decimal

- $\quad$ Modelo II: SWEAT

$k-0,58 \cdot X+0,25 \cdot X+0,16 \cdot X(\mathrm{~W} / \mathrm{m} \mathrm{C})$

onde:

$\mathrm{X}$ = fração mássica de água

$\mathrm{X}$ = fração mássica de carboidrato

$\mathrm{X}=$ fração mássica de gordura

Pela análise dos valores da Tabela 6, percebe-se que o erro é bastante alto e variado na determinação teórica da condutividade térmica, levando os valores sempre para cima (desvio positivo).

TABELA 6 - Erro calculado para condutividade térmica, segundo equações (VIII) e (IX)

\begin{tabular}{cccccc}
\hline & \multicolumn{3}{c}{$\mathrm{W} / \mathrm{m}^{\circ} \mathrm{C}$} & \multicolumn{3}{c}{$\%$} \\
\cline { 2 - 6 } Ensaios & $\mathrm{K}_{\text {calc } \mid \text { expl }}$ & $\mathrm{k}_{\text {equachio } \mathrm{VII}}$ & $\mathrm{k}_{\text {equacio } \mathrm{IX}}$ & Erro $_{\text {equacio } \mathrm{VII}}$ & Erro $_{\text {equacio } \mathrm{XX}}$ \\
\hline 1 & 0,3412 & 0,5416 & 0,5616 & $\mathbf{5 8 , 7 5}$ & 64,59 \\
2 & 0,5504 & 0,5365 & 0,5564 & 2,53 & 1,09 \\
3 & 0,3055 & 0,4969 & 0,5264 & 62,64 & 72,30 \\
4 & 0,3753 & 0,4923 & 0,5216 & 31,16 & 38,99 \\
5 & 0,3546 & 0,5158 & 0,5407 & 45,45 & 52,48 \\
6 & 0,3331 & 0,5158 & 0,5407 & 54,84 & 62,32 \\
7 & 0,3561 & 0,5158 & 0,5407 & 44,84 & 51,84 \\
8 & 0,2486 & 0,5192 & 0,5442 & 108,84 & 118,90 \\
9 & 0,3182 & 0,4865 & 0,5177 & 52,90 & 62,71 \\
10 & 0,4164 & 0,5124 & 0,5373 & 23,06 & 29,03 \\
11 & 0,4976 & 0,5500 & 0,5676 & 10,52 & 14,06 \\
\hline Erro = (valor teórico - valor experimental)/ valor experimental x 100
\end{tabular}

\section{4 - CONCLUSÕES}

As equações obtidas, excetuando-se a viscosidade, apresentaram bons resultados, permitindo que, uma vez conhecida a composição do suco, desde que os componentes variem dentro da faixa estudada, pode-se a princípio simular as propriedades termofisicas sem a necessidade de medi-las experimentalmente. Estas equações de modo geral visam prever as propriedades termofisicas de sucos tropicais, otimizando processos industriais, como dimensionamento dos equipamentos utilizados no processamento de alimentos.

A viscosidade aumentou com adição de carboidrato (elevação da variável $\mathrm{X}_{2}$ ). A condutividade térmica teve comportamento oposto em relação a essa variável, diminuindo com aumento de $\mathrm{X}_{2}$. Muito pouco foi possível comentar sobre a variável $\mathrm{X}$ (\% de gordura em relação à água e ao carboidrato), pois esta, por estar em pouca quantidade, não representou tanta variação sobre as variáveis de resposta, provocando falta de ajuste de algumas propriedades termofisicas (viscosidade e densidade).

Os modelos encontrados na literatura em geral predizem bem os dados experimentais.

\section{5 - REFERÊNCIAS BIBLIOGRÁFICAS}

[1] ALVARADO, J.D. Nota. Viscosidad y energía de activación de jugos filtrados, Revista Española de Ciencia y Tecnologia de Alimentos, v. 33, n. 1, p. 8793, 1993.

[2] BARROS, B.; SCARMINIO,I. S.; BRUNS, R. E. 1995. Planejamento e otimização de experimentos. Metodologia de superfície de resposta., Editora da Unicamp.

[3] BAYINDIRLI, L. Mathematical Analysis of Variation of Density and Viscosity of Apple Juice with temperature and Concentration, Journal of Food Processing and Preservation. v. 16, p. 23-28, 1992.

[4] CONSTENLA, D.T.; LOZANO, J.E.; CRAPISTE, G.H. Thermophysical Properties of Clarified Apple Juice as a Function of Concentration and Temperature, Journal of Food Science, v. 54, n. 3, p. 663-668, 1989.

[5] KOLAROV, K.M., GROMOV, M.A. Khromitelna Promishenost v. 2, n. 10, p. 32, In: JOWITT, R., ESCHER, F., HALlSTROM, H. F., MEFFERT, Th., SPISS, W.E.L., VOS, G. Physical Properties of Foods. Applied Science Publisher, NY, 1973. Apud: VIRENDRA, K.B. SINGH, A.K., SINGHY. Prediction of Thermal Conductivity of Fruit Juices by Thermal Resistence Model, Journal of Food Science, v. 54, n. 4, p. 1007-1012, 1989.

[6] MIN, D. B.; THOMAS, E. L. Application of response surface analysis in the formulation of whipped topping, Journal of Food Science, v. 45, p. 346348, 1980

[7] MOURA, S.C.S.R.; FRANÇA, V.C.L.; LEAL, A.M.C.B.. Propriedades termofisicas de soluções modelo similares a sucos Parte I, Ciênc. Tecnol. Aliment., v. 23, n. 1, p. 6268, jan.-abr., 2003.

[8] PEACOCK, S. Predicting physical properties of factory 
juices and syrups, International Sugar Journal, v. 97, n. 1162, p. 571-577, 1995.

[9] POLLEY, S.L.; SNYDER, O.P.; KOTNOUR, P. A compilation of Thermal Properties of Foods, Food Technology, v. 34, n. 11, p.76-94, 1980.

[10] SWEAT, V.E. Thermal Porperties of Foods. In: RAO, M.A., RIZVI, S.S.H. Engineering Properties of Foods. Marcel Dekker Inc, NY, 1986. Apud: VIRENDRA, K.B. SINGH, A.K., SINGHY. Prediction of Thermal Conductivity of Fruit Juices by Thermal Resistence Model, Journal of Food Science, v. 54, n. 4, p. 1007-1012, 1989.

[11] TELIS-ROMERO ,J.; TELIS, V.R.N.; GABAS, A.L.; YAMASHITA, F. Thermophysical Properties of Brazilian Orange Juice as Affected by temperature and Water Content, Journal of Food Engineering, v. 38, p.27-40, 1998.
[12] VAN WAZER, J.R.; LYONS, J.W.; KIM, K.Y.; COLWELL, R.E. Viscosity and Flow Measurement - A Laboratory Handbook of Rheology. New York: Intersience Publishers, 1972. p.215-230.

[13] VIRENDRA, K.B.; SINGH, A.K.; SINGHY. Prediction of Thermal Conductivity of Fruit Juices by Thermal Resistance Model, Journal of Food Science, v. 54, n. 4, p. 1007-1012, 1989.

\section{6 - AGRADECIMENTOS}

Ao CNPq pela concessão das bolsas de Iniciação Científica - PIBIC. 